\title{
A posição de amamentar determina o aparecimento do trauma mamilar?*
}

\author{
DOES BREASTFEEDING POSITION INFLUENCE THE ONSET OF NIPPLE TRAUMA? \\ ¿LA POSICIÓN DEL AMAMANTAMIENTO DETERMINA LA APARICIÓN DEL TRAUMA DEL \\ PEZÓN?
}

\author{
Kelly Pereira Coca', Mônica Antar Gamba², Rebeca de Sousa e Silva ${ }^{3}$, \\ Ana Cristina Freitas de Vilhena Abrão ${ }^{4}$
}

\section{RESUMO}

O estudo buscou identificar as variáveis de posicionamento e pega, durante a amamentação, relacionadas aos traumas mamilares. Estudo caso-controle que investigou o aparecimento do trauma mamilar entre mulheres internadas em um hospital Universitário de São Paulo, em 2004 e 2005. Os casos foram puérperas com diagnóstico de trauma mamilar uni ou bilateral. Para a análise dos dados, foram aplicados os testes qui-quadrado, $\mathrm{t}$ de Student, razão de chances (IC=95\%) e análise de correspondência. Foram estudadas 146 puérperas e seus recém-nascidos, sendo 73 casos e 73 controles. As variáveis de posicionamento e pega, estatisticamente significativas para a ocorrência da lesão, foram: criança com pescoço torcido, queixo longe da mama e lábio inferior virado para dentro. A prevenção do trauma, no início da amamentação, é decisiva para a continuidade desta prática. O acompanhamento do posicionamento adequado é determinante para o estabelecimento da amamentação efetiva e prolongada.

\section{DESCRITORES}

Aleitamento materno.

Mamilos.

Estudos de casos e controles.

Saúde da mulher.

Enfermagem obstétrica.

\section{ABSTRACT}

The aim of the study was to identify the breast feeding position and holding variables related to nipple trauma. This casecontrol study assessed the onset of nipple trauma among women hospitalized at a University Hospital in the city of São Paulo, in 2004 and 2005. Subjects were puerperae diagnosed with unilateral or bilateral nipple trauma. Data analysis was performed using chi-square, Student's t, and odds ratio tests $(\mathrm{Cl}=95 \%)$ and correspondence analysis. Participants were 146 puerperal women and their newborns, being 73 cases and 73 controls. Statistically significant position and holding variables for causing lesions were the following: newborns with their necks bent/contorted, chin away from the breast and lip-related defect (turned inward). Trauma prevention at the beginning of breast feeding is crucial for continuing this practice. Following adequate positioning is decisive for establishing effective and prolonged breast feeding.

\section{KEY WORDS}

Breast feeding.

Nipples.

Case-control studies.

Women's health.

Obstetrical nursing.

\section{RESUMEN}

El estudio buscó identificar las variables de posición y agarre, durante el amamantamiento, relacionadas a los traumas del pezón. Se trata de un estudio de caso y control que investigó el aparecimiento del trauma del pezón en mujeres, internadas en un hospital Universitario de San Pablo, en 2.004 y 2.005. Los casos fueron puerperales, con diagnóstico de trauma del pezón unilateral o bilateral. Para el análisis de los datos, fueron aplicadas las pruebas chi-cuadrado, $t$ de Student, razón de probabilidades $(\mathrm{IC}=95 \%)$ y análisis de correspondencia. Fueron estudiadas 146 mujeres en el estado puerperal y sus recién nacidos, siendo 73 casos y 73 controles. Las variables de posición y agarre, estadísticamente significativas para la ocurrencia de la lesión, fueron: niño con el cuello torcido, mentón lejos del pezón de la mama e labio inferior virado para dentro. La prevención del trauma, en el inicio del amamantamiento, es decisiva para la continuidad de esta práctica y el acompañamiento de la posición adecuada es determinante para el establecimiento del amamantamiento efectivo y prolongado.

\section{DESCRIPTORES}

Lactancia materna.

Pezones.

Estudios de casos y controles.

Salud de la mujer.

Enfermería obstétrica.

\footnotetext{
* Extraído da dissertação "Traumas mamilares: estudo dos fatores associados", Departamento de Enfermagem, Universidade Federal de São Paulo, 2005 ${ }^{1}$ Enfermeira obstetra. Mestre em enfermagem obstétrica. São Paulo, SP, Brasil. kcocaepm@hotmail.com ${ }^{2}$ Professora Doutora do Departamento de Enfermagem da Universidade Federal de São Paulo, São Paulo, SP, Brasil. monica@denf.epm.br ${ }^{3}$ Professora Doutora do Departamento de Medicina Preventiva da Universidade Federal de São Paulo, São Paulo, SP, Brasil. rebeca@medprev.epm.br ${ }^{4}$ Professora Doutora do Departamento de Enfermagem da Universidade Federal de São Paulo, São Paulo, SP, Brasil.anaabrao@denf.epm.br

$\begin{aligned} \text { Rev Esc Enferm USP } & \text { Recebido: 22/08/2007 } \\ 2009 ; \text { 43(2):446-52 } & \text { Aprovado: } 16 / 04 / 2008\end{aligned}$
}




\section{INTRODUÇÃO}

O aleitamento materno (AM) é uma prática que deve se constituir em uma estratégia de promoção à saúde e requer compreensão dos determinantes associados à sua interrupção. Evidências científicas têm demonstrado que o aleitamento materno exclusivo é a melhor forma de alimentar as crianças, pois se constitui em base para o desenvolvimento biológico e emocional ${ }^{(1)}$, além de proporcionar proteção contra infecções do primeiro ano de vida e diminuição das doenças crônicas, durante a idade adulta(2).

Estudos realizados no Brasil urbano mostraram que a prevalência do aleitamento exclusivo está muito abaixo do recomendado, indicando que $53,1 \%$ das crianças na faixa etária entre 0-30 dias de vida, estavam recebendo somente leite materno, ocorrendo, a partir daí, uma brusca redução nos índices para 21,6\%, na faixa etária entre 91-120 dias, e 9,7\%, entre 151-180 dias, com duração média de 23 dias de aleitamento materno exclusivo e 296 dias de aleitamento materno não exclusivo(3).

No Estado de São Paulo, o índice de aleitamento materno exclusivo em crianças nos primeiros quatro meses raramente alcançou níveis superiores a $30 \%$. Os fatores de risco identificados para essa situação foram a baixa escolaridade materna, ausência do Programa Hospital Amigo da Criança, primiparidade e maternidade precoce ${ }^{(4)}$.

Estes resultados coincidem, em parte, com estudo realizado em um ambulatório de aleitamento materno com 100 mulheres e crianças atendidas, no período de outubro de 2001 a julho de 2003, com as mesmas características acima descritas. Nestes casos, o aleitamento materno exclusivo esteve presente, em torno do terceiro e quarto mês, em apenas $36 \%$ das mulheres ${ }^{(5)}$. Quando realizada a pri- meira consulta, em média, entre o sétimo e décimo dia de vida da criança, $23 \%$ delas já estavam recebendo água, chás ou leite artificial. Além dessas intercorrências identificadas, outras também contribuíram para o desmame precoce, dentre elas, o trauma mamilar (TM) e o uso de chupetas(5).

A lesão mamilar pode ser definida como uma solução de continuidade da pele do mamilo e/ou aréola, ou seja, por fissura, escoriação, erosão, dilaceração e vesículas, o que dificulta o processo de amamentação por ocasionar desconforto e dor ${ }^{(6)}$.

Dentre as causas mencionadas para ocorrência dos traumas mamilares, é possível identificar características sóciodemográficas, relacionadas à gestação, às mamas, ao parto, a fatores neonatais e à amamentação(6).

Vale destacar que, durante a amamentação, o posicionamento, a preensão do mamilo e sucção do leite pela criança são fatores fundamentais para a ocorrência do tipo de trauma. Vários autores referem que, ao amamentar, a posição inadequada da criança e a apreensão incorreta da região mamilo-areolar podem desencadear o aparecimento de lesões mamilares; porém, são poucas as pesquisas que comprovam tal afirmação(4,7-9). A sucção inadequada também é descrita como uma das causas dos traumas podendo, se corrigida, ser um fator de proteção(9-14).

Em relação às características do posicionamento adequado da criança diante da mama, ela deve estar com o seu corpo próximo e voltado para a mãe, as nádegas apoiadas, a cabeça e o corpo alinhados com a boca, na mesma altura da mama, em frente à aréola ${ }^{(9,15)}$ (Figura 1). A correta apreensão da região mamilo-areolar é um passo importante para o início da mamada. A criança deve estar com os lábios voltados para fora, a boca bem aberta, as bochechas arredondadas e o queixo tocando o peito da mãe ${ }^{(7)}$ (Figura 2).

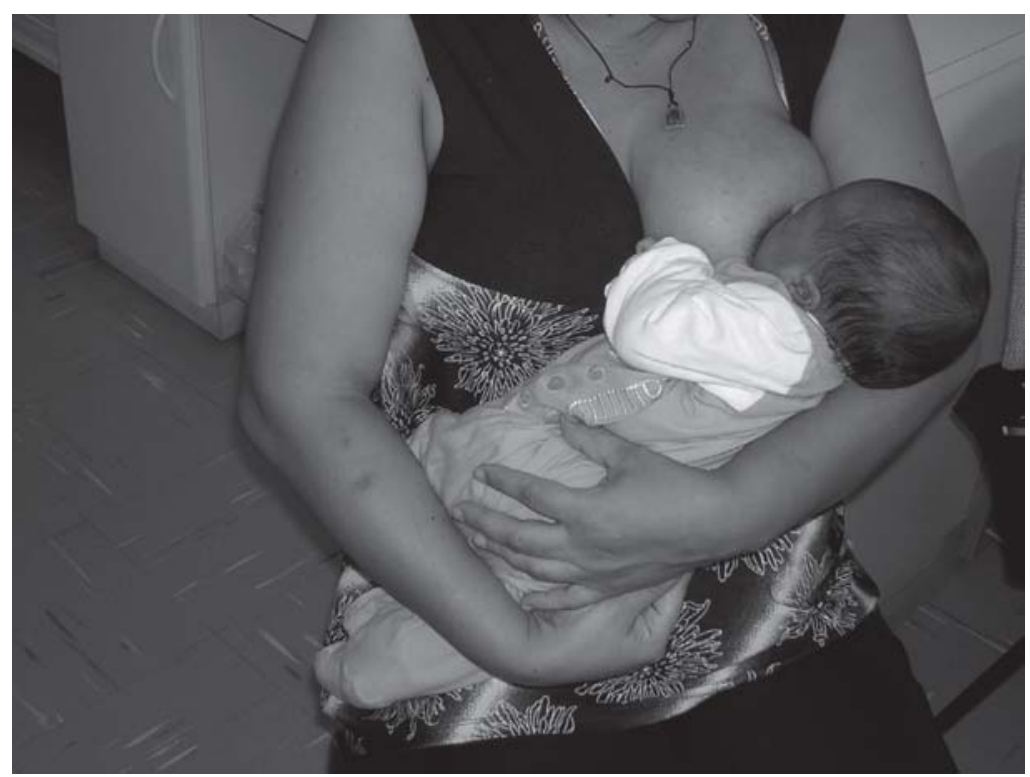

Figura 1 - Posicionamento adequado da criança durante a amamentação. 


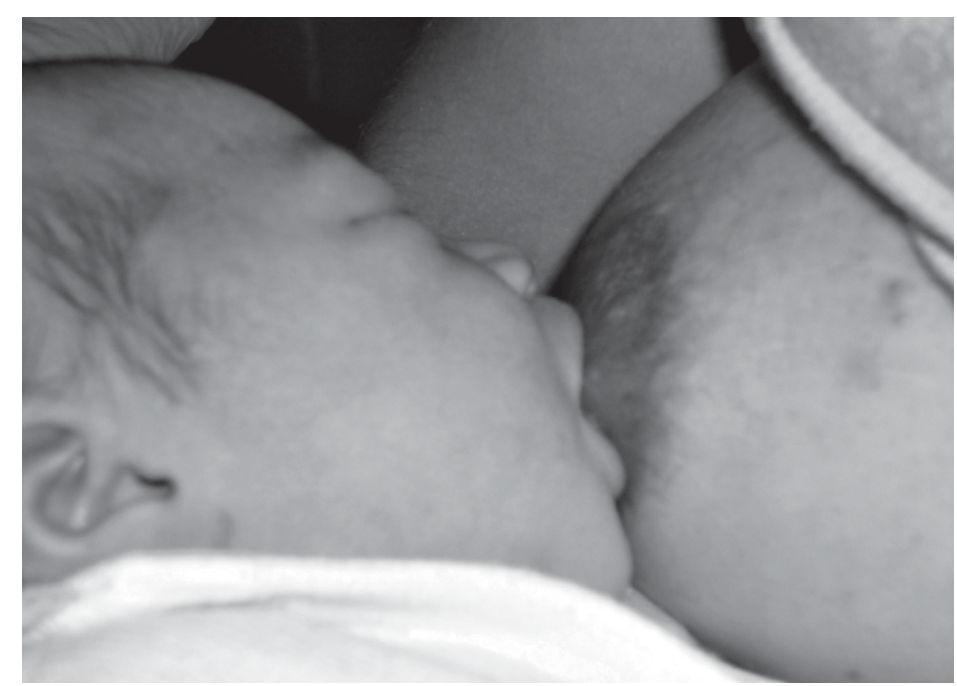

Figura 2 - Pega adequada da criança durante a amamentação

A sucção eficiente está relacionada a uma boa pega, podendo ser considerada uma ação preventiva para as lesões mamilares, alteração da arcada dentária, síndrome do respirador bucal, da deglutição atípica e das alterações fonoarticulatórias ${ }^{(16)}$.

Acreditando que ações preventivas podem contribuir para minimizar as dificuldades na amamentação, a base dessa investigação foi identificar fatores relacionados à posição da criança durante a amamentação e apreensão do mamilo para o desenvolvimento de traumas mamilares.

\section{OBJETIVO}

Identificar as variáveis relacionadas ao posicionamento e apreensão durante a amamentação e o aparecimento de traumas mamilares.

\section{MÉTODO}

Trata-se de um estudo epidemiológico, do tipo casocontrole, o qual permite conhecer a exposição aos supostos fatores de risco estudados nos quais há suspeita de relação com a doença. Inicia-se pela identificação e seleção dos doentes e, em seguida, estabelece controles comparáveis.

Neste estudo, considerou-se como caso a puérpera com trauma mamilar uni ou bilateral, durante a primeira semana pós-parto e em aleitamento materno. Caracterizou-se como tendo trauma mamilar a mulher que apresentasse uma solução de continuidade da pele na região mamilo-areolar ${ }^{(6)}$. 0 grupo controle foi selecionado mediante a ausência de trauma uni ou bilateral, de forma a ser semelhante aos casos, segundo idade e dia de puerpério, por serem possíveis fatores de confusão. Foi selecionado um controle para cada caso.

Foram incluídas todas as puérperas e os recém-nascidos internados nas enfermarias de Alojamento Conjunto de uma Unidade de Obstetrícia, no período compreendido entre setembro de 2004 a maio de 2005 e que estavam em processo de amamentação. Foram excluídas da amostra mulheres que estavam amamentando mais de um filho, quando o filho apresentasse dificuldade persistente para sucção e apreensão da região mamilo-areolar e aquelas cujas crianças apresentassem malformações da região de palato e língua, além das mulheres que estavam amamentando somente em uma das mamas (mastectomia unilateral).

Assim sendo, a população de estudo constituiu-se de 146 binômios (mãe-filho), sendo 73 casos e 73 controles.

A coleta de dados iniciou-se após a aprovação do Comitê de Ética em Pesquisa da Universidade Federal de São Paulo (CEP no 0785/04) e a assinatura do Termo de Consentimento Livre e Esclarecido. Diariamente, era feito um levantamento das mulheres internadas na Unidade e realizada a seleção daquelas que iriam participar da pesquisa. Realizou-se a entrevista, na qual foram coletados dados referentes às características das puérperas (idade, escolaridade, gestação, paridade, dia de puerpério e tipo de parto) e características das crianças pesquisadas (sexo, peso ao nascer e idade gestacional). Posteriormente, foram realizados o exame físico e observação direta da técnica de amamentação. As variáveis relacionadas à posição durante a mamada foram: posição da mãe, posição da criança em relação à mãe, posição da criança, queixo da criança em relação à mama da mãe, boca da criança, lábio inferior da criança, língua da criança, posição da boca em relação à aréola, sucção e deglutição da criança(16). Para esta avaliação, foi feita a observação da mamada, por especialista, no momento em que a mesma ocorria, acompanhando desde seu início até o término ou interrupção espontânea. Todos os dados coletados foram registrados em um formulário específico, construído especialmente para este estudo.

Para análise estatística aplicaram-se os testes qui-quadrado e $t$ de Student. Para a verificação da presença de associações estatisticamente significativas $(p=\leq 0,05)$ entre os possíveis fatores e o trauma mamilar, foram calcula- 
dos, na análise univariada, a razão de chances (intervalo com 95\% de confiança) e análise de correspondência para associação conjunta entre as variáveis relacionadas à posição de amamentação com ocorrência da lesão.

\section{RESULTADOS}

Foram estudadas 146 puérperas e seus respectivos recém-nascidos, distribuídos em 73 casos e 73 controles. As mulheres apresentaram, em média, idade de 26 anos ( $p=$ $0,591 ;$ SD 6,0$)$, oito anos de estudo ( $p=0,263$; SD 2,7) e estavam no segundo dia de puerpério. Quanto aos dados obstétricos, 32\% eram primigestas ( $p=0,215), 35 \%$ eram primíparas $(p=0,385)$ e $64 \%$ tiveram partos vaginais $(p=0,002)$. As características das crianças estudadas foram: peso, ao nascer, em média 3100 gramas ( $p=0,418) ; 52 \%$ eram do sexo masculino $(p=0,508)$ e $92 \%$ nasceram a termo $(p=0,347)$.

Quanto ao posicionamento do binômio durante a mamada, observou-se: a mãe com ombros tensos e/ou debru- çada sobre a criança $(p=0,501)$; a criança posicionada distante da mãe $(0,071)$. Estas variáveis não foram estatisticamente significativas para o aparecimento da lesão. Entretanto, a posição da criança desalinhada foi estatisticamente significativa para o aparecimento do trauma mamilar $(p=0,047)$.

No que se refere aos aspectos relacionados à apreensão do mamilo foram observadas as variáveis: criança com boca pouco aberta e apontando para frente $(p=0,279)$, língua não visível $(p=0,071)$, aréola visível mais abaixo da mama $(p=0,863)$, sucção rápida $(p=0,319)$ e deglutição não audível $(p=0,596)$ não foram estatisticamente significativas para o aparecimento da lesão mamilar. Quando a criança apresentou o queixo distante da mama e o lábio voltado para dentro, os achados explicaram o trauma, pois foram significativos ( $p$ respectivamente $=0,044$ e 0,001).

Após análise univariada dos dados, foram estimados os valores das razões de chances (odds ratio) e respectivos intervalos com 95\% de confiança (Tabela 1).

Tabela 1 - Resultados das associações entre trauma mamilar e posicionamento do binômio e apreensão do mamilo, com os Odds ratio, respectivos intervalos de confiança e p descritivo: estudo caso-controle grupo - São Paulo - 2006

\begin{tabular}{|c|c|c|c|c|c|c|}
\hline Variável & Caso & Controle & $\mathbf{p}$ & OR & \multicolumn{2}{|c|}{ IC $(95 \%)$} \\
\hline \multicolumn{7}{|c|}{ Posição da mãe ao amamentar } \\
\hline Adequada & 41 & 45 & & & & \\
\hline Inadequada & 32 & 28 & 0,501 & 1,254 & $(0,648$ & $2,428)$ \\
\hline \multicolumn{7}{|c|}{ Posição da criança em relação à mãe } \\
\hline Adequada & 61 & 68 & & & & \\
\hline Inadequada & 12 & 05 & 0,071 & 2,675 & $(0,891$ & $8,030)$ \\
\hline \multicolumn{7}{|c|}{ Posição da criança } \\
\hline Adequada & 31 & 43 & & & & \\
\hline Inadequada & 42 & 30 & $0,047^{*}$ & 1,942 & $(1,006$ & $3,749)$ \\
\hline \multicolumn{7}{|c|}{ Queixo da criança } \\
\hline Adequada & 60 & 68 & & & & \\
\hline Inadequada & 13 & 05 & $0,044^{*}$ & 2,947 & $(0,992$ & $8,749)$ \\
\hline \multicolumn{7}{|l|}{ Boca da criança } \\
\hline Adequada & 48 & 54 & & & & \\
\hline Inadequada & 25 & 19 & 0,279 & 1,480 & $(0,726$ & $3,017)$ \\
\hline \multicolumn{7}{|c|}{ Lábio inferior da criança } \\
\hline Adequada & 38 & 60 & & & & \\
\hline Inadequada & 35 & 13 & $0,001^{*}$ & 4,251 & $(1,998$ & $9,047)$ \\
\hline \multicolumn{7}{|c|}{ Língua da criança } \\
\hline Adequada & 17 & 27 & & & & \\
\hline Inadequada & 56 & 46 & 0,071 & 1,934 & $(0,940$ & $3,978)$ \\
\hline \multicolumn{7}{|c|}{ Observação da aréola } \\
\hline Adequada & 46 & 47 & & & & \\
\hline Inadequada & 27 & 26 & 0,863 & 1,061 & $(0,540$ & $2,883)$ \\
\hline \multicolumn{7}{|c|}{ Sucção da criança } \\
\hline Adequada & 56 & 60 & & & & \\
\hline Inadequada & 17 & 12 & 0,319 & 1,518 & $(0,666$ & $3,460)$ \\
\hline \multicolumn{7}{|c|}{ Deglutição da criança } \\
\hline Adequada & 64 & 66 & & & & \\
\hline Inadequada & 09 & 07 & 0,596 & 1,326 & $(0,466$ & $3,773)$ \\
\hline
\end{tabular}

* significância estatística 
A mulher cuja criança foi amamentada com pescoço torcido, queixo distante da mama e lábio inferior voltado para dentro teve a chance de 1,942 vezes (IC 95\%=1,006 a 3,749 ), 2,947 vezes (IC $95 \%=0,992$ a 8,749 ) e 4,251 vezes (IC $95 \%=1,998$ a 9,047), respectivamente, de apresentar trauma mamilar quando comparada à mãe com a ausência dessas características.
Na análise de associação, observou-se que as características da mãe com trauma mamilar foram: mãe com ombros tensos e/ou deitada sobre o recém-nascido, criança com pescoço torcido, língua da criança não visível, apresentando sugadas rápidas, criança com lábio inferior voltado para dentro e boca pouco aberta, além de uma maior proporção da aréola abaixo da boca do recém-nascido (Figura 3).

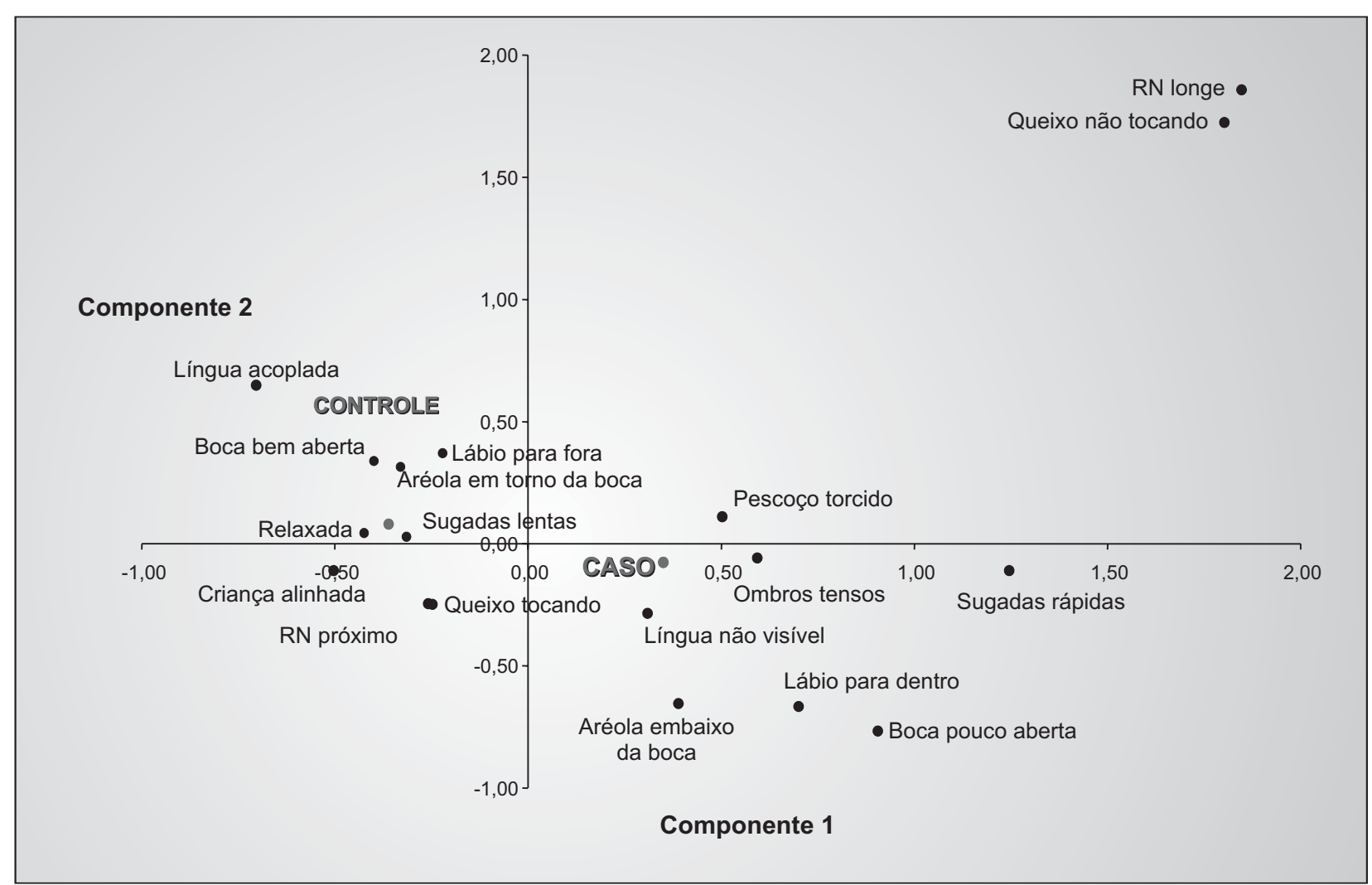

Figura 3 - Análise de correspondência de fatores associados a traumas mamilares quanto ao posicionamento da criança e apreensão do mamilo durante a amamentação: estudo caso - controle - São Paulo - 2006

\section{DISCUSSÃO}

O estudo permitiu identificar alguns fatores associados ao posicionamento e à pega, durante a mamada, que podem contribuir para a ocorrência de lesão mamilar.

A lesão mamilar esteve presente até o sexto dia pósparto, ocorrendo, em média, no segundo dia. O objetivo de controle dessa variável foi possibilitar oportunidade para que todos os controles pudessem vir a se tornarem casos. Dessa forma, todas as puérperas do grupo controle tiveram chance de apresentar lesão mamilar. Os resultados confirmaram o que a literatura tem demonstrado sobre o período de ocorrência na primeira semana pós-parto ${ }^{(6)}$.

No que se refere à escolaridade das mulheres estudadas, observou-se que, em ambos os grupos apresentaram, em média, oito anos de estudo. Portanto, nessa investigação, o nível de escolaridade não foi importante para o aparecimento da lesão mamilar. Autores acreditam que um maior nível de escolaridade aumenta a chance de uma nutriz amamentar com sucesso, pois referem que a amamentação não é instintiva e sim uma prática que pode ser aprendida $^{(17)}$.

Quanto ao número de gestações, não foram encontrados estudos que demonstrassem uma relação direta com a presença dos traumas mamilares. Nessa pesquisa, encontrou-se uma maior proporção de primigestas com lesão mamilar (57,4\%). Quanto à paridade, a maior incidência de lesões mamilares tem sido verificada em mulheres primíparas, considerando a inexperiência na técnica de amamen$\operatorname{tar}^{(18)}$. Neste presente estudo, observou-se que $54,9 \%$ de primíparas apresentavam lesão mamilar, enquanto $45,1 \%$ delas não a apresentavam.

Em relação ao tipo de parto, estudos identificam maior incidência de trauma mamilar em mulheres submetidas a parto cesárea ${ }^{(15)}$. Acredita-se que isto ocorra porque a dor e o repouso, por vinte e quatro horas após o parto, dificul- 
tam o posicionamento adequado da criança ao ser amamentada. Acrescenta-se o fato da mulher receber anestésicos que podem interferir no comportamento de sucção da criança ${ }^{(19)}$. Atualmente, recomendações como deambulação precoce, estímulo à diferentes posições de amamentação e precaução com relação ao tipo e dosagem dos anestésicos favorecem a amamentação.

Em relação às características neonatais, observa-se que a criança do sexo masculino apresenta sucção mais vigorosa, segundo relatos de puérperas e referências de alguns estudos. Porém, nesta avaliação, não foi identificada diferença entre os grupos estudados. Tais resultados também foram encontrados em outro estudo ${ }^{(20)}$. O peso das crianças estudadas foi, em média, de 3.100 gramas, tanto para o grupo controle como para os casos. Quanto à idade gestacional, um estudo identificou que a maior incidência de traumas ocorre em puérperas com recém-nascidos entre 37 e 40 semanas $^{(18)}$, no entanto, resultados inversos foram observados para mães de prematuros. A sucção do recém-nascido prematuro pode apresentar menor força de sucção, resultando em menos solicitação do tecido mamilar, bem como adiamento das primeiras mamadas para estabilização clínica ${ }^{(20)}$. No entanto, este estudo não identificou diferenças entre os grupos.

Quanto à avaliação da amamentação, estudou-se a posição da mãe e da criança, preensão mamilo-areolar, sucção e deglutição. Recomenda-se que a posição durante a amamentação seja confortável para a mãe e para o bebê. A mãe deve estar relaxada, com as costas apoiadas, podendo ficar sentada ou deitada. Quanto ao posicionamento do bebê, sua cabeça deve estar em linha reta em relação ao seu corpo, próximo da mãe e de frente para o peito(16).

No que se refere ao posicionamento do binômio, durante a amamentação, observou-se que as posições inadequadas da mãe e criança não foram estatisticamente significativas para o aparecimento da lesão. Já quanto à posição da criança, com o pescoço torcido, o resultado foi significativo quando comparado entre os grupos. A mulher, cujo filho estava posicionado de forma inadequada, teve 1,942 vezes a chance (IC 95\%=1,006 a 3,749) de apresentar trauma quando comparada àquela cujo filho estava posicionado adequadamente.

Para uma preensão correta, a mãe deve posicionar os lábios da criança no mamilo, com o objetivo de estimular o reflexo de busca, procura e preensão. Em seguida, ela deve aproximar a criança da mama. A criança deve apreender parte ou toda a região mamilo-areolar, de forma que a sua boca permaneça bem aberta, o queixo toque a mama, o lábio inferior fique voltado para fora, a língua esteja sobre a gengiva, observando-se parte da aréola, sendo mais acima da boca que abaixo dela. Lábios apertados e apontando para frente indicam pega inadequada ${ }^{(16)}$. Neste estudo, a boca pouco aberta, a língua não visível e aréola abaixo da boca não foram significativas para o aparecimento do trauma.

Quanto à posição do queixo da criança, os resultados foram estatisticamente significativos $(p=0,044)$, demons- trando ser um importante fator para ocorrência da lesão mamilar. Os resultados mostraram ainda que a puérpera, cujo filho estava posicionado de forma que seu queixo não tocava a mama, teve 2,947 vezes a chance (IC $95 \%=0,992$ a 8,749 ) de desenvolver trauma, quando comparada àquela cuja criança estava posicionada de forma adequada.

No que se refere à posição do lábio da criança, autores indicam que, durante a preensão da mama, o lábio inferior da criança deve estar virado para fora, caracterizando uma preensão correta ${ }^{(16)}$. A posição do lábio inferior durante a mamada foi estatisticamente significativa $(p=0,001)$, demonstrando que $72,9 \%$ das crianças, cujas mães pertenciam ao grupo de casos, estavam com o lábio inferior voltado para dentro. Pode-se observar ainda que a nutriz, cuja criança estava com a posição do lábio inadequada, teve 4,251 vezes a chance (IC 95\%=1,998 a 9,047) de apresentar trauma quando comparada àquela cujo filho apresentava lábios voltados para fora.

Durante a amamentação, a sucção é lenta e profunda e pode-se ver e/ou ouvir a deglutição. Quando a criança apresenta sugadas rápidas e faz barulhos altos de chupar, significa que a técnica está incorreta ${ }^{(16)}$. A sucção não eficiente é reconhecida como fator que predispõe ao trauma mamilar ${ }^{(18)}$. A variável sucção não se mostrou significativa neste estudo, contrapondo-se aos resultados encontrados na literatura.

No que se refere à deglutição, esta variável foi identificada para ser analisada em conjunto com a variável sucção, pois não tem relação com a ocorrência do trauma mamilar. Os resultados encontrados não mostraram diferença estatisticamente significativa. Estas variáveis, apesar de não serem significativas, aproximaram-se do limite para a identificação do aparecimento dos traumas. Quando analisadas em conjunto, estes resultados sinalizam para a necessidade de outras investigações.

A análise de correspondência possibilitou avaliar o comportamento das variáveis de posicionamento e pega, em relação ao aparecimento da lesão. A puérpera e a respectiva criança apresentaram características inadequadas quanto à observação da mamada, ao passo que o binômio dos controles apresentou características consideradas adequadas durante a amamentação (Figura 1).

Esses achados permitem identificar aspectos relevantes que corroboram com dados da literatura no que se refere aos fatores intervenientes para o desmame precoce de lactentes, contribuindo com a prática clínica.

\section{CONCLUSÕES}

Por meio dos resultados obtidos, é possível concluir que as variáveis posição da criança-com pescoço torcido, queixo da criança - distante da mama e lábio da criança - voltado para dentro foram estatisticamente significativas para a ocorrência da lesão mamilar. O acompanhamento do po- 
sicionamento da criança, no início da amamentação, foi determinante para a prevenção do trauma e para a continuidade desta prática.

Os resultados encontrados permitem ainda apontar subsídios científicos que embasem a técnica de amamentação, prevenindo a incidência de traumas mamilares, con- siderados responsáveis por inúmeros casos de desmame precoce.

Diante do exposto, ressalta-se a importância da observação e do aconselhamento da puérpera durante o processo da amamentação, como parte integrante da assistência à saúde da mulher.

\section{REFERÊNCIAS}

1. Lucas A, Fewtrell MS, Davies PS, Bishop NJ, Clough H, Cole TJ. Breastfeeding and catch-up growth in infants born small for gestational age. Acta Paediatr. 1997;86(6):564-9.

2. World Health Organization. WHO Collaborative Study Team on the Role of Breastfeeding on the Prevention of Infant Mortality: effect of breastfeeding on infant and child mortality due to infectious diseases in less developed countries: a pooled analysis. Lancet. 2000;355(9202):451-5.

3. Brasil. Ministério da Saúde. Secretaria de Políticas de Saúde. Área de Saúde da Criança. Prevalência do aleitamento materno nas capitais brasileiras. Brasília; 2001.

4. Venancio SI, Escuder MML, Kitoko P, Rea MF, Monteiro CA Freqüência e determinantes do aleitamento materno em municípios do Estado de São Paulo. Rev Saúde Pública. 2002;36(3):313-8

5. Abrão ACFV. Aleitamento materno: seguimentos e controles [resumo]. In: Anias do 2을 Congresso Paulista de Bancos de Leite Humano e 12을 Encontro Paulista de Aleitamento Materno; 2003 set. 11-14; Marília, SP, Brasil.

6. Vinha VHP. Traumas Mamilares (ferimentos): prevenção e cuidados. In: Vinha VHP. O livro da amamentação. São Paulo: CLR Balieiro; 1999. p. 45-54.

7. Biancuzzo M. Sore nipples: prevention and problem-solving. Herndon: WMC Worldwide; 2000. [Simplifying Breastfeeding Management Self-Learning Series].

8. Novak FR, Almeida JAG, Silva RS. Casca de banana: uma possível fonte de infecção no tratamento de fissuras mamilares. J Pediatr (Rio J).2003;79(3):221-6.

9. Huml S. Sore nipples: a new look at an old problem through the eyes of a dermatologist. Pract Midwife. 1999;2(2):28-31.

10. Giugliani ERJ. O aleitamento materno na prática clínica. J Pediatr (Rio J). 2000;76 Suppl 3:S238-52.
11. Pugh LC, Buchko BL, Bishop BA, Cochran JF, Smith LR, Lerew DJ. A comparison of topical agents to relieve nipple pain and enhance breastfeeding. Birth. 1996;23(2):88-93.

12. Pietschnig B, Pani M, Kafer A, Bauer Wais E, Lischka A. Use of soft laser in the therapy of sore nipples in breastfeeding women. Adv Exp Med Biol. 2000;478:437-8.

13. Smith JW, Tully MR. Midwifery management of breastfeeding: using the evidence. J Midwifery Womens Health. 2001;46(6):423-38

14. Brent N, Rudy SJ, Redd B, Rudy TE, Roth LA. Sore nipples in breast-feeding women: a clinical trial of wound dressings vs conventional care. Arch Pediatr Adolesc Med. 1998;152(11):1077-82.

15. Teruya K, Serva VB. Manejo da lactação. In: Rego JD. Aleitamento Materno. São Paulo: Atheneu; 2002. p. 113-30.

16. Cordeiro MT. Postura, posição e pega adequadas: um bom início para a amamentação. In: Rego JD, editor. Aleitamento materno. São Paulo: Atheneu; 2002. p. 131-55.

17. Lawrence RA, Lawrence RM. Management of the motherinfant nursing couple. In: Lawrence RA, Lawrence RM Breastfeeding: a guide for the medical profession. $6^{\text {th }}$ ed. St. Louis: Mosby; 2005. p. 255-316.

18. Shimoda GT, Silva IA, Santos JL. Características, freqüência e fatores presentes na ocorrência de lesão de mamilos em nutrizes. Rev Bras Enferm. 2005;58(5):529-34.

19. Vinha VHP, Shimo AKK, Scochi CGS, Pelá NTR. A sucção não eficiente como causa de trauma mamilar e pseudohipogalactia. Femina. 1986;14(8):740-3.

20. Peres JAT, Santana RM, Lacordia PL, Dolnikoff M, Deláscio D. Uso da lisozima na fissura da Mama puperpural. J Brás Ginecol. 1980;90(6):317-9. 